\title{
EXTERNAL, INTERNAL AND SEMI-INTERNAL VIBRATIONS IN MOLECULAR SOLIDS: SPECTROSCOPIC CRITERIA FOR IDENTIFICATION*
}

\author{
Paras N. PRASAD and Raoul KOPELMAN \\ Department of Chemisiry. The University of Michigan, Ann Arbor, Michigan 48104, USA \\ Received 28 February 1973 \\ Revised manuscript received 6 June 1973
}

\begin{abstract}
A new method to identify the nature of vibrations in molecular crystals as external or intemal is proposed. The criterion is that in isotopic mixed crystals of protonated and corresponding perdeuterated compounds, the external motions (phonons) are in the amalgamation limit, while the interna modes, and the "semi-internal" methyl torsions, are usually in the separated band linit. This criterion is sapported by experimental isotopic mixed crystal studies of many molecular crystals. Previous criteria, like temperature and isotope shifts, are shown to be less reliable.

Anthracenc and biphenyl Raman spectra, as.well as literature data, are used as illustrations.
\end{abstract}

\section{Introduction}

In molecular crystals, the vibrations are usually classified [1] as: (i) internal vibrations which are essentially intramolecular motions and subject to solid state interactions only within the limits of perturbation, (ii) external vibrations which correspond to the motion of the molecule as a whole and are entirely solid state effects because in free molecular states these motions have zero vibrational frequency. Also in some molecular crystals, motions like torsions exist which cannot strictly be classified as internal or external vibrations [2]. Though they are intramolecular motions and have barniers which are intramoleculat in origin, they very often have a large contribution to the barrier from the solid state interaction. We thus classify them in category (iii) semi-internal vibrations.

To identify internal vibrations, the criterion often used is that these vibrations are little affected by solid state interactions and have roughly the same frequency as they have in the gas or even in solution. This correlation is very often used to identify the intramolecular mode. This, however, is not always useful. There are systems like biphenyl which do not have the same geometry in the gas phase [3] (where the two benzene

\footnotetext{
* Supported by NIH Grant NS08116.
}

rings are not planar) and in the room temperature solid phase (where the two benzene rings seem to be planar [4]). The correlation may not be of much help in such cases. Another criterion [5] used is that intramolecular modes in the solid phase are temperature inderendent. This has been often used to distinguish a low lying in. tramolecular mode from lattice modes. Recently we have found that in many systems (durene, anthracene, biphenyl) the low lying intramolecular modes are temperature dependent. The temperature dependence criterion can thus be misleading.

Another distinction between internal and semiinternal modes, and the external mode is based on isotope shifts. These are mostly piedictable and generally much smaller for external vibrations (phonons). This distinction is obviously part of the general use of isutope shifts in the identification of normal modes. Specifically, the semi-internal methyl group torsions are usually identified on the basis of isotope shift. The ratio of the perproto methyl torsion frequency and the corresponding perdeutero methyl torsion frequency in a simple model is 1.41 which corresponds to the ratio of the square roots of the moments of inertia of the $\mathrm{CH}_{3}$ and $\mathrm{CD}_{3}$ groups. We have encountered signiticant deviations [6] (1.3 compared to 1.4 ) in this value and in oux opinion, this criterion has to be used with care. 
Another possible criterion, though rarely used, is the magnitude of the crystai bandwidth relative to the basic excitation energy. Intramolecular modes (internal and semi-internal) will usually have much smaller relative bandwidths than phonons, due to the relatively smaller intermolecular interaction. This is the ieason why intramolecular modes are usually classified as vibrational excitons, while phonons rarely are $[2,7]$. In practice bandwidths are usually studied only indirectly, via the Davydov components [8], and, in addition to problems ol assignment, some crystals, like those with one molecule per unit cell, have only one Davydov component, thus not enabling any estimate of the bandwidth.

In this paper we propose a new method of distinction between internal and semi-internal modes, and the external mode. It is based on an "experimental amplification" of some of the above criteria, specifically combining the following two: the relative bandwidth and the isotope shift. It is well known that for an isotopic mixed crystal one gets two limiting cases. I. The separated bund limit [8], for cases where the bandwidth is small compared to the isotope shift ("trap depth"). II. The amalgamation limit [9], for cases where the isotope shift is small compared to the bandwidth. We also note that in isotopic mixed crystals the bandwidth is casily revealed, thus avoiding some of the problems associated with Davydov components in neat crystals.

Our method is also supported by experimental isotopic mixed crystal studies of many molecular crys. tals: dimethylacerylene [10], benzere [7], durene [2], hexamethylbenzene [6], bipheny] (ref. [11] and reported in this paper), naphthalene $[8,9]$, anthracene (reported in this paper) and pherianthrene [12]. From these investigations we developed the criterion that in isotopic mixed molecular crystals of protonated and corresponding perdeuterated compounds, the lattice motions (phonons) are in the amalgamation limit [9], while the internal motions and the semi-internal methyl torsions are usually in the separated band limit $[2,8]$.

We have also tested the above criterion on low lying Raman active intramolecular modes of durene, anthra. cene and biphenyl, where a significant temperature dependence has been observed. It again seems to work well in these cases.

\section{Experimental}

Single crystals (neat and isotopic mixed) grown from the melt in a capillary under vacuum were used for the experiments. Regulated flow of cold nitrogen vapor provided a variable temperature bath from room temperature down to $100^{\circ} \mathrm{K}$. To get lower temperatures $\left(25^{\circ} \mathrm{K}\right)$ helium vapor was used. The spectra were recorded on a Ranalog Spex double spectrometer model 1401 using 4880 A laser emission from a Coherent Radiation Laboratory argon ion laser for excitation. The spectral resolution was $1 \mathrm{~cm}^{-1}$.

\section{Results and discussion}

The temperature dependence study shows that the phonon frequencies shift about 10-20\% in going from room temperature to $100^{\circ} \mathrm{K}$. The sharpening is the most remarkable feature. In most of the cases the factor group splittings ( 5 to $20 \mathrm{~cm}^{-1}$ ) on the Raman phonons are not even resolved at room temperature but they are usually resolved at $100^{\circ} \mathrm{K}$. Such temperature dependence studies on phonon states already exist in the literature. The paper by Ito et al. [13] describes the temperature dependence of Raman active phonons in many molecular crystals. Usually, the change from $100^{\circ} \mathrm{K}$ to lower temperature is small. The durene crystal, however, seems to be an exception. Here, though the phonon frequencies do not shift that much in going from $100^{\circ} \mathrm{K}$ to $25^{\circ} \mathrm{K}$, the line-width decreases very significantly. Some features which are not resolved at $100^{\circ} \mathrm{K}$ get very well resolved at $25^{\circ} \mathrm{K}$. De. tails of the temperature dependence study on durene are reportecl elsewhere $[2,14]$. Here we would simply like to empiasize that this difference in temperature dependence of the vibrational frequency and that of its line-width arises because they are usually not caused by the same anharmonic interaction. The line broadening is caused by two mechanisms $[15,16]$ : (i) The lifetime broadening due to relaxation effects. This involves anharmonic processes in which the vibration decays into two or more other vibrations conserving both the momentum (wave vector) and energy. (ii) Fluctuation broadening which does not involve any vibrational relaxation but a scattering mechanism: the broadening is not caused by decay of the vibration but only due 
to scattering of this vibration with other vibrations and involves quartic anharmonicity terms. On the other hand, the temperature dependent frequency shift, is caused by anharmonic interactions which do not require energy conservation. Also, the thermal expansion of the lattice contributes only to the frequency shift but not to the line broadening.

The internal modes have been assumed in the past to be temperature independent. We have seen temperature dependent frequency shifts on one internal mode in durene, on two internal modes in anthracene and on three internal modes in biphenyl. The temperature shifts of the two anthracene fundamentals and of the three biphenyl fundamentals are shown in figs. 1 and 2 . The temperature dependence of the durene vibration is reported in detail elsewhere [14]. In the case of durene the $272 \mathrm{~cm}^{-1}$ fundamental of durene- $h_{14}$ shows an increase of $9 \mathrm{~cm}^{-1}$ in going from room temperature to $100^{\circ} \mathrm{K}$. In the case of anthracene- $h_{14}$ the mode at $285 \mathrm{~cm}^{-1}$ increases by $6 \mathrm{~cm}^{-1}$ while the fundamental at $242 \mathrm{~cm}^{-1}$ increases by $3 \mathrm{~cm}^{-1}$ in going from room temperature to $100^{\circ} \mathrm{K}^{\ddagger}$. Biphenyl shows an interesting temperature dependence. The mode at $250 \mathrm{~cm}^{-1}$ (biphenyl- $h_{10}$ value) decreases in frequency

\% Bree and Kydd [17] have obseryed temperature dependence on the corresponding anthracene- $d_{10}$ modes near the melting point.

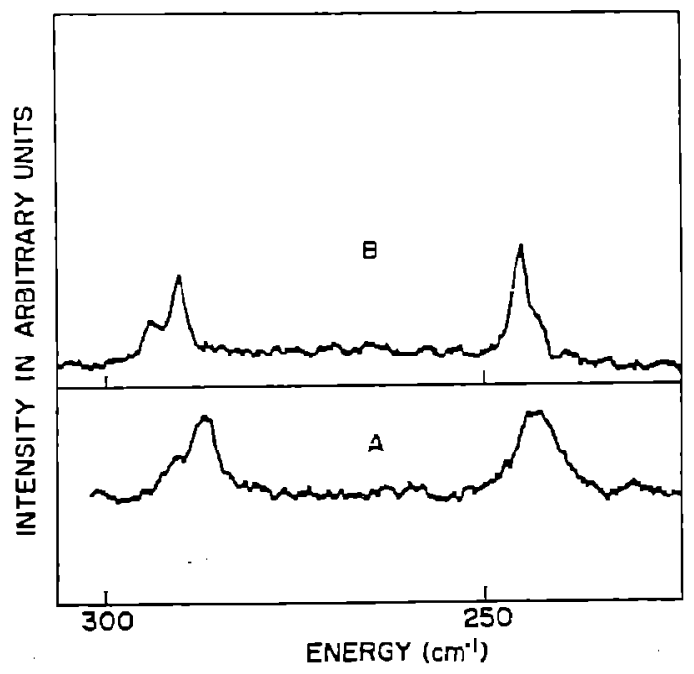

Fig. 1. Raman spectra of neat anthracene- $h_{10}$ crystal: $A$ is the spectrum at room temperature, $B$ that at $100^{\circ} \mathrm{K}$. The resolution is $1 \mathrm{~cm}^{-1}$. by $5 \mathrm{~cm}^{-1}$ while the one at $330 \mathrm{~cm}^{-1}$ increases by $5 \mathrm{~cm}^{-1}$ going from room temperature to $100^{\circ} \mathrm{K}$. Also the mode at $1149 \mathrm{~cm}^{-1}$ of biphenyl th 10 shows a decrease of $4 \mathrm{~cm}^{-1}$ for the same temperature change The corresponding perdeutero compounds show similar features. In a recent paper [14] we have discussed the origin of this temperature dependent shift in durene and from our various considerations we have suggested that the major contribution to the temperature shift does not come from the thermal expansion of the lattice. Anharmonic interactions which exist even in a lattice without thermal expansion may often dominate over the contribution due to thermal expansion. We thus see that the temperature dependence criterion for distinguishing between external and internal modes seems to be unreliable, if not misleading.

For semi-internal motions, like methyl torsion, very often the following criterion is used: the ratio of the per proto frequency to the corresponding perdeutero frequency is 1.41 , which corresponds to the square root of the ratio of the moments of inertia of the $\mathrm{CH}_{3}$

1: Pasquier [18] has also observed temperature dependence on biphenyl modes. While he repoits that almost every intra.molecular band in the biphenyl Raman spectrum is temperature dependent, we see significant temperatura shifts on only three intramolecular fundamentals.

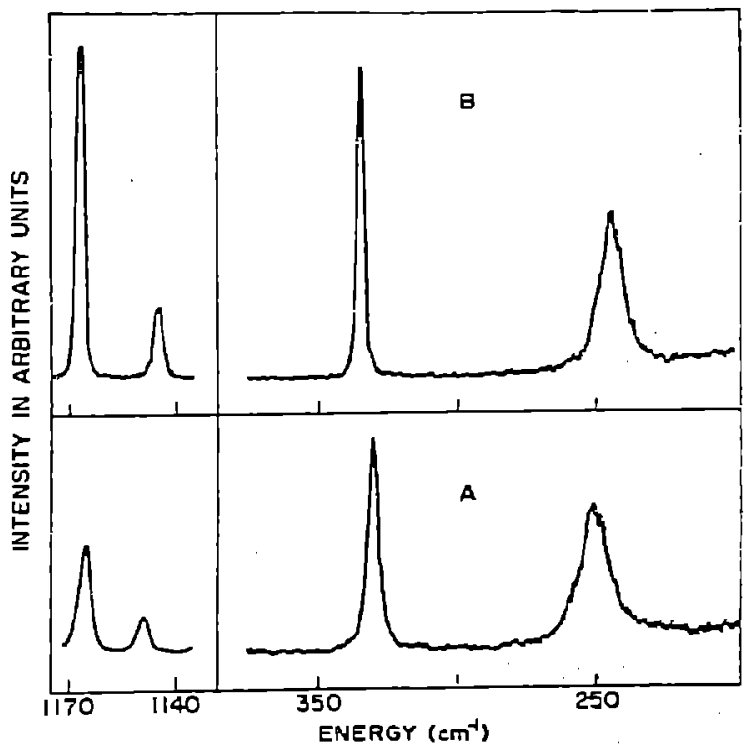

Fig. 2. Raman spectra of neat biphenyl $h_{10} \mathrm{crj}$ stal: $A$ is the spectrum at room temperature, B tlat at $100^{\circ} \mathrm{K}$. The resalution is $1 \mathrm{~cm}^{-1}$. 
and $\mathrm{CD}_{3}$ groups. This is true in a simple minded picture where the methyl groups are assumed to be rotating against a molecular unit of infinite moment of inertia. Calculations have been made considering the moment of inertia of the molecular unit rotating against the methyl group and a value of 1.40 has been obtained in the case of hexamethylbenzene [19]. Furthermore, the intermolecular interaction can alter this value substantially. As a matter of fact, we find this ratio to be only 1.32 in the case of hexamethylbenzene [6]. Also in durene [2] this ratio is 1.37. In hexa. methylbenzene we think that this deviation is caused by interaction of methyl torsions with phonons [6].

In isotopic mixed crystals the phonons obey the amalgamotion limit in which the frequency shifts continuously from one component (perproto) to another (perdeutero) as one changes the concentration of the perdeutero compounds [9]. A typical spectrum obtained is shown in fig. 3 which is obtained for anthracene iso topic mixed crystals at $100^{\circ} \mathrm{K}$. The reason that phonons are usually in the amalgamation limit is that the isotopic perturbation on the lattice motion is small compared to the lattice-dynamical interactions. For most intramolecular modes, both internal and semiinternal (but not, perhaps, for biphenyl ring torsion, vide infra), the isotopic perturbation is large. In the exciton theory formalism this means that the isotopic frequency shift (trap-depth) is large compared to the vibrational exciton bandwidth. For this reason these modes are in the separated band limit. In other words, if one makes a $50 \%$ crystal solution of perproto and perdeutero compounds, one will see transitions due to both perproto and the corresponding perdeutero compound. Fig. 4 shows such a study on the temperature dependent intramolecular modes of biphenyl. We clcarly sec that the middle spectrum is a superposition of the top and bottom spectra which correspond, respectively, to biphenyl- $h_{10}$ and biphenyl- $d_{10}$. We have tested this criterion also in dimethylacetylene [10], benzene [7], naphthalene [8,9], hexamethylbenzene [6] and phenanthrene [12] and it seems to work well.

The distinction between internal modes and semiinternal modes is made by their dissimilarity. The semiinternal modes (methyl torsions) are very anharmonic and show large temperature broadening and shift while the internal modes are sharp. The methy! torsions, which we have classified as semi-internal motions, show

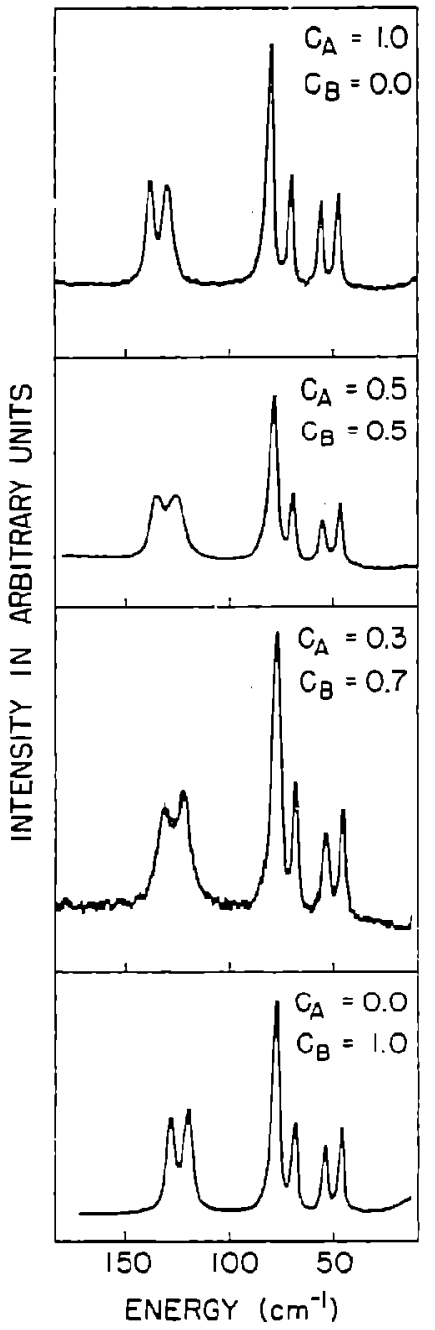

Fig. 3. Raman spectra of reat and isotopic mixed anthraccne crystals at $100^{\circ} \mathrm{K}$ and at a resolution of $1 \mathrm{~cm}^{-1}: C_{\mathrm{A}}$ is the concentration of anthracene-1 $1_{10}, C_{B}$ that of anthracene-d 10 .

great resemblance to phonons so far as line broadening and temperature dependence of the frequency shift are concemed. In durene, for example [2], the transitions due to methyl torsions are so broad at room tem. perature that they can hardly be seen. Even at $100^{\circ} \mathrm{K}$ they are so broad that the Davydov splitting of $10 \mathrm{~cm}^{-1}$ on the $195 \mathrm{~cm}^{-1}$ torsion of durene- $h_{14}$ (and the 142 $\mathrm{cm}^{-1}$ torsion of durene- $d_{14}$ ) cannot be seen. With significant decrease in line-width following the lowering of the remperature, this Davydov splitting is clearly resolved at $25^{\circ} \mathrm{K}$ [2]. A similar line sharpening is seen 


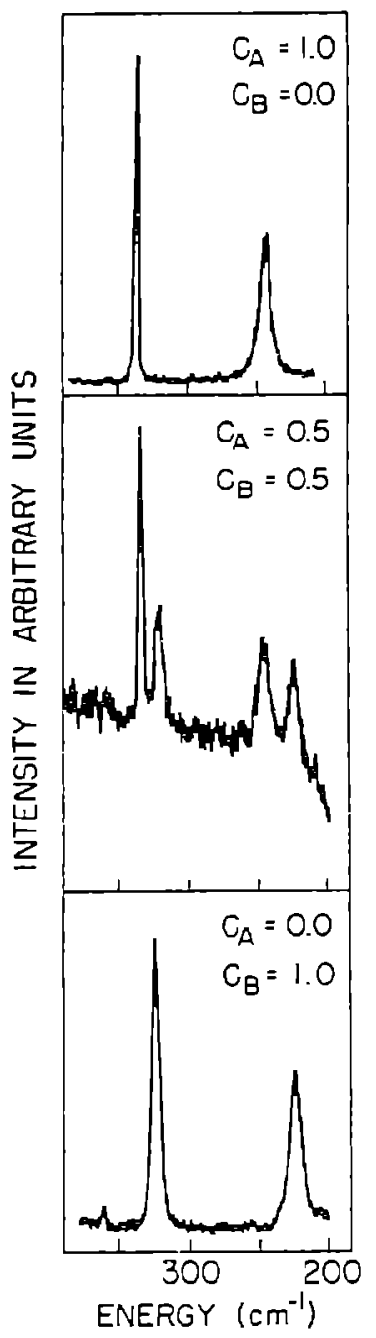

Fig. 4. Raman spectra of neat and isotopic mixed biphenyl crystals at $100^{\circ} \mathrm{K}$ and at a resolution of $1 \mathrm{~cm}^{-1} ; C_{\mathrm{A}}$ is the concentration of biphengl- $h_{10}, C_{\mathrm{B}}$ that of biphenyl- $d_{10}$.

in the case of the hexame thylbenzene torsion [6].

Because of the broadening the methyl torsions might be confused with phonon overtones or combinations as the lowest methyl torsions usually lie in that range. Our criterion proves to be helpful in this concern as methyl torsions are in the separated band limit while the phonons are in the amalgamation limit. This criterion has been of great assistance in the investigations of durene [2] and hexamethylbenzene [6].

At the end, we would like to point out that our criterion depends only on the condition that for internal and semi-internal modes the isotopic shift (trap- depth) is large compared to the exciton band width. This is almost invariably the case with the hydrocarbons because the deuteration shifts of vibrational frequencies are large. In systems like hexachlorobenzene, however, there is no hydrogen and isotopic substitution of $35 \mathrm{Cl}$ by ${ }^{37} \mathrm{Cl}$ will involve a small frequency shift. Also, semiinternal motions like biphenyl ring torsions, which have small isotopic shifts, may be in the amalgamation limit (we are currently working on it). For these cases our criterion may not work (neither for any C-13 shifts). In view of these limitations we suggest that our method should not always be taken as the sole criterion but be used judiciously or as complementary to other criteria of identifying the nature of the vibration.

\section{Acknowledgement}

We thank the National Science Foundation for grant GP-8345, which enabled the departmental pu:clase of the Ramalog.Spex double spectrometer.

\section{References}

[1] G. Venkataraman and V.C. Sahni, Rev. Mlod. Phys. 24 (1970) 409.

[2] P.N. Prasad and R. Kopelman, J. Chem. Phỵs. 58 (1973) 126.

[3] O. Bastiansen, Acta Chem. Scand. 3 (1949) 408.

[4]. G. Robertson, Nature 191 (1961) 593; J. Trotter, Acta Cryst. 14 (1961) 1135 ; A. Horgreaves and S.H. Rizvi, Acta Cryst. 15 (1962) 365.

[5] A. Hadri, B. Wyncike, G. Morlot and X. Gerbaus, J. Chem. Phys. 51 (1969) 3514.

[6] P.N. Prasad, S.D. Woodruff and R. Kopelnan, Chem. Phys. 1 (1973) 173.

[7] H.K. Hong and R. Kopelman, J. Chem. Fnys. 58 (1973) 384.

[8] P.N. Prasad and R. Kopelman, J. Chem. Phys. 57 (1972) 856, and references therein.

[9] P.N. Prasad and R. Kopelman, J. Chem. Phys. 57 (1972) 863 , and references therein.

[10] P.N. Ptusad and R. Kopelman, Chem. Phys. Letters 20 (1973) 513.

[11] P.S. Friedman, R. Kopelman and P.N. Prasad, unpublished work.

[12] P.N. Prasad and R. Kopelman, unpublished work.

[13] M. Ito, M. Suzuki and T. Yokoyama, in: Excitons, magnons and phonons in molecular crystals, ed. A.B. Zahian (Cambridge Univ. Press, London, 1968) p. 1.

[14] P.N. Prasad and R. Kopelman, J. Chem. Phys. (June 1, 1973), to be published. 
[15] K.K. Rebane, Impurity spectra of solids, translated by J.S. Shier (Plenum Press, New York, 1970).

[16] R.M. Hochstrasser and P.N. Prasad, in: Excited states, Vol. 1. ed. E.C. Lim (Academic Press, New York), to be published.
[17] A. Bree and R.A. Kydd, Chem. Phys. Letters 3 (1969) 357.

[18] B. Pasquier, Mol Cryst. Liquid Cryst. 11 (1970) 35.

[19] M. Gerard, G. Dumas and J. Michel, Compt. Rend. Acad. Sci. (Paris) 272B (1971) 836. 